\title{
Study on Extraction Method of Overhead Power Line Based on Prior Knowledge with Single visible light Camera
}

\author{
Wenyu Wang ${ }^{1, a, *}$,Yuanjing Liu ${ }^{2, b}$, Xianpei Wang ${ }^{2, c}$ \\ ${ }^{1}$ School of Journalism and Communication, Wuhan Sports University, Wuhan 430073, Hubei Province \\ ${ }^{2}$ Electronic Information School, Wuhan University, Wuhan 430072, Hubei Province \\ a405424059@qq.com,b2419795725@qq.com,,xpwang@whu.edu.cn \\ *Corresponding author
}

Keywords: Overhead Power Line, Extraction, UAV

\begin{abstract}
UAV equipped with a simple visible light camerato obtain the depth information of power line and the ground through monocular or binocular vision is the focus of the research. And how to use digital image processing method to extract the power linerapidly, accurately, automatically is one of the core issues. Therefore, in this paper, three methods of power line extraction are analyzed, namely, the Hough transform, Radon transform and LSD algorithm, pointing out the problems existing in the three algorithms. Aiming at the problems, a method based on priori knowledge-restrained scope, was proposed, in order to improve the accuracy of the power line detection. The effectiveness of the algorithm is verified by the experiments.
\end{abstract}

\section{Summary of line extraction}

In target identification, linear feature extraction is a very important link, at the same time, it has been the focus of many experts ${ }^{[1]}$, The commonly used linear extraction methods can be divided into the following three categories generally ${ }^{[2][3][4]}$. The first category is the line extraction method based on transform domain, which is performed in the transform domain space. Through the relationship between transform domain and image domain, the straight line is locatedby inverse transform, the classical algorithms are Hough transform and Radon transform; the second category is based on gradient.Combingthe amplitude and direction of gradient, the straight line is extractedby"edge region growing", such as Line Segment Detector (LSD) ${ }^{[5]}$, Phase grouping etc.; the third category is line extraction method based on chain code. Use the linear feature of digital image to track the edge chain code and realize the line extractionaccording to the characteristics of chain code ${ }^{[6]}$. In this paper,power line extraction effect of Hough transform, Radon transform and LSD algorithm are compared and analyzed, problems are pointed out and a kind of algorithm which restraints on power line extraction range using a priori knowledge is put forward, in order to increase the accuracy rate of power line detection. Related experiments show that this algorithm is effective.

\section{Classical line extraction algorithm}

Fig. 1 is collected by small UVA with a camera. Based on MATLAB programming environment, this paper uses Sobel algorithm for edgedetection. Fig. 2 (a) is the extraction result of Hough transform, fig. 2 (b) is the extraction result of Radon transform, fig. 2 (c) is the extraction result of LSD transform.

Although Hough transform line detection method has better anti-noise ability, when background is a more complex, such as farmland, houses, trees weeds etc., its performance greatly decreases under the interference of the background, sometimes it can not detect the power line completely, otherwise, some unrelated line segments are wrongly extracted, such ashouse eaves, ridge, weeds and so on. The anti-noise ability of Radon transform stillexists even if the background is complex . However, the classical Radon transformcan only obtain the linear direction and radial coordinate, it 
is unable to determine the location coordinates, the starting point and end point of the line segment, and line width information. As is seen from the LSD detection results ,this method shows great performance in dealing with details, even if the power line is weak. In terms of the error rate, LSD have better performance than Hough transform, and the ability of locating power line is also good, but it also detects some small segments in the background by mistake, sothe characteristics of power line in the image can be used to make further improvement.

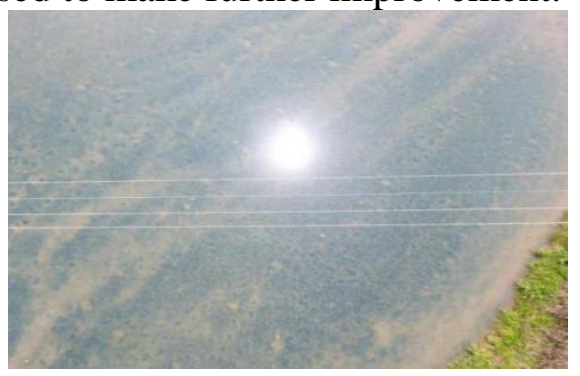

Fig. 1 Picture representative

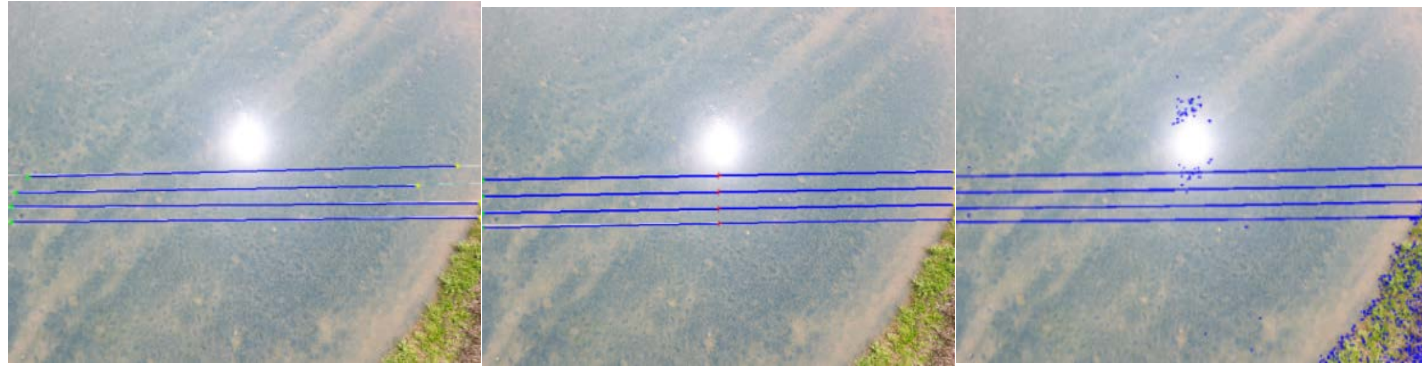

(a) Extraction results of HT (b) Extraction results of RT (c) Extraction results ofLSD

Fig. 2 The extraction results of three kinds of classic transformation

\section{Based on prior knowledge power line extraction}

For a particular area of the power lines, the shooting routes can be planned in advance, and pictures are taken along the power line corridor, then the power line can be extracted based on the information of previously extracted power line, which are shoot in the same area.

Based on this idea, this paper presents a method to limit the range of power line extraction. The method cannot only avoid error extraction of the power line caused by the river, house and other complex background to a large extent, but also reduce the time of power line extraction.

The central idea of this approach is that: for the power line image group collected along the same power line corridor, first determine the maximum value $Y_{\max }$ and minimum value $Y_{\min }$ of the power line of one of the photos based on the fact that it has been precisely extracted, (the power line is usually in the horizontal direction in the image), so the power lines of the remaining pictures in the same image group can be extracted in the range of $\left(\left(Y_{\min }-y_{t h}\right),\left(Y_{\max }+y_{t h}\right)\right)$.

The flow of the method is:

(1) Find the range of power lines $\left(Y_{\min }, Y_{\max }\right)$ on the basis of an image of a power line that has been extracted.

(2) Considering the instability factors during the UAV shooting, $y_{\text {th }}$ can be estimated.

(3) In the next images in the same image group, on the basis of the edge detection, perform Radon transform in the range of $\left(\left(Y_{\min }-y_{t h}\right),\left(Y_{\max }+y_{t h}\right)\right)$, find out the information of extreme point, and convert it to linear space. At last, draw a straight line.

\section{Experiment and result analysis}

In order to verify the efficiency of the power line extraction method based on prior knowledge, 
first let the UAV fly along the power line and acquire the aerial power line image group (Fig. 3 (a) (b) (c)), then acquire the power line information one of the image (Fig. 3 (a)). Based on MATLAB R2014a platform, use therange based prior knowledge proposed in this paper to test Hough transform and Radon transform respectively (all based on Sobel edge detection).

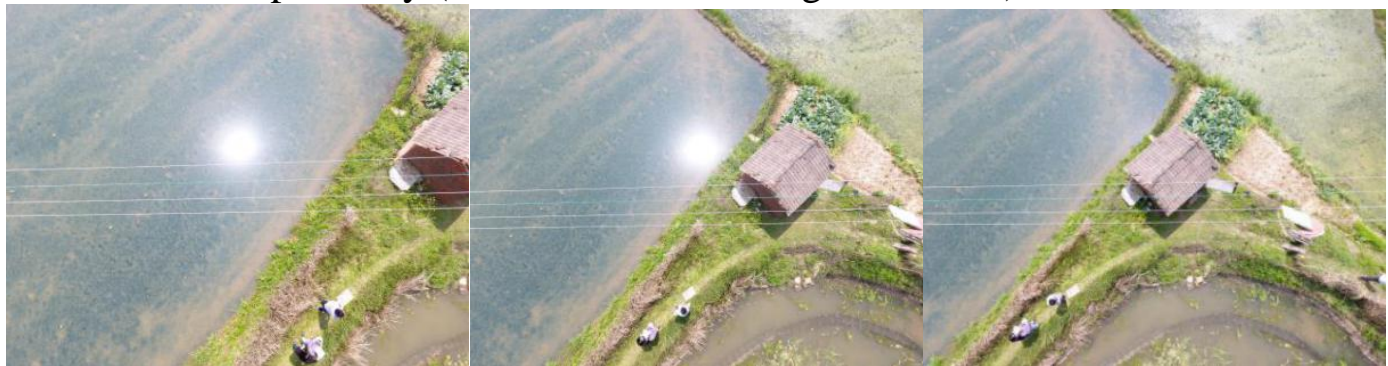

(a) (b) (c)

Fig. 3 A group of images taken along a power line

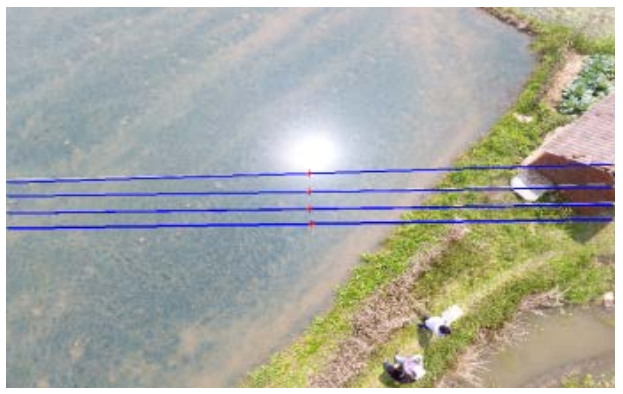

Fig. 4 Pre-extraction of fig. 3 (a)

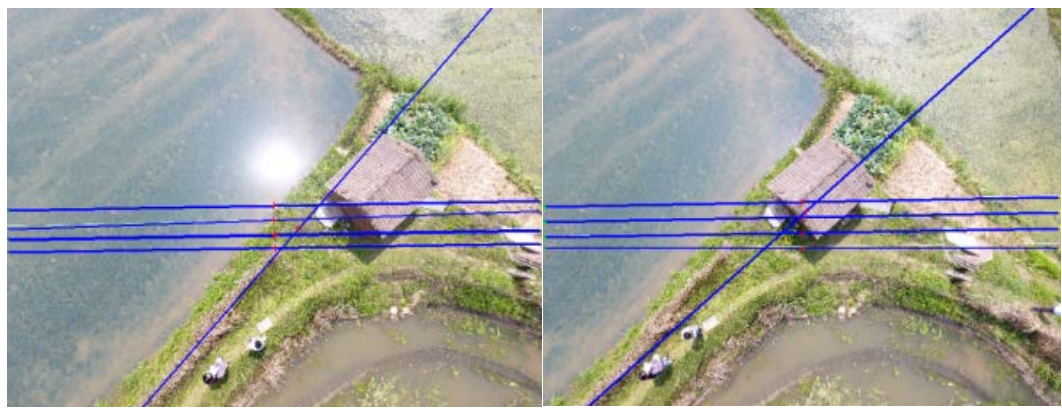

(b) Extraction results of fig. 3 (b) (c) Extraction results of fig. 3 (c)

Fig. 5 Power line extraction results of Radon transform of fig. 3 (b) (c) without constraints

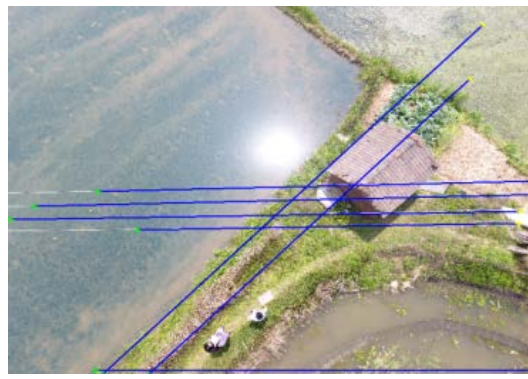

(b) Extraction results of fig. 3 (b)

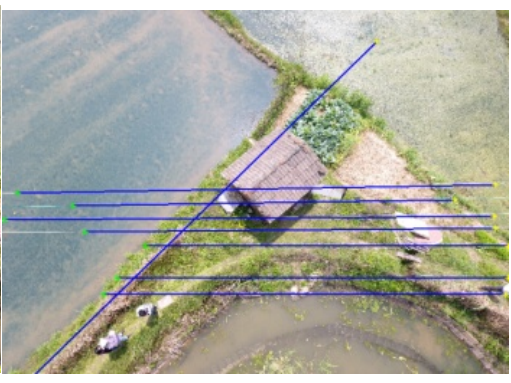

(c) Extraction results of fig. 3 (c)

Fig. 6 Power line detection results of Hough transform of fig. 3 (b) (c) without constraints 


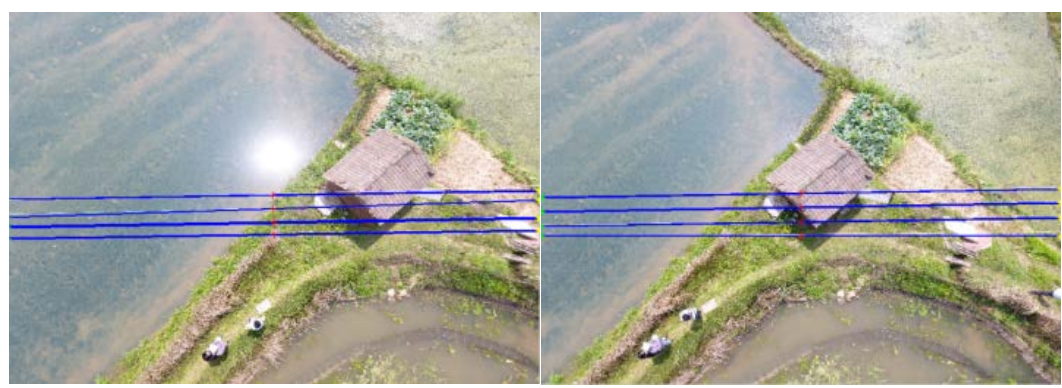

(b) fig. 3 (b) Extraction results

(c) fig. 3 (c) Extraction results

Fig. 7 Power line extraction results of Radon transform of fig.3 (b) (c)with constraints

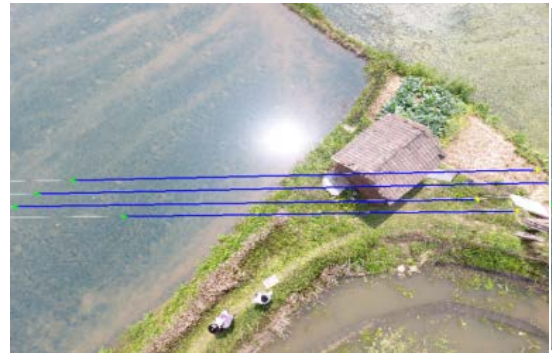

(b) fig. 3 (b) Extraction results

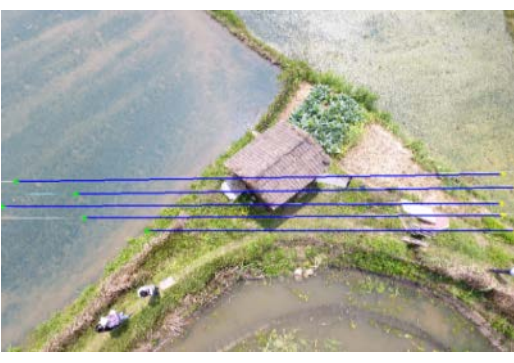

(c) fig. 3 (c) Extraction results

Fig. 8 Power line extraction results of Hough transform of fig. 3 (b) (c) with constraints

Fig. 5 shows the result of Radon transform with an unregulated threshold, which is in the absence of prior knowledge. Fig. 6 shows the result of Hough transform with an unregulated threshold. From the above, the power lines can be wrongly extracted because of the interference of farmland or other background.

Table 1 Comparison of the error detection rate of the two cases

\begin{tabular}{|cc|c|c|c|c|}
\hline \multirow{2}{*}{$\begin{array}{l}\text { Error } \\
\text { detection rate }\end{array}$} & \multicolumn{2}{|c|}{ There is no constraint } & \multicolumn{2}{|c|}{ There are constraints } \\
\hline Condition & $\begin{array}{c}\text { Radon } \\
\text { Transform }\end{array}$ & $\begin{array}{c}\text { Hough } \\
\text { Transform }\end{array}$ & $\begin{array}{c}\text { Radon } \\
\text { Transform }\end{array}$ & $\begin{array}{c}\text { Hough } \\
\text { Transform }\end{array}$ \\
\hline \multicolumn{2}{|c|}{ Fig.3 (b) } & $20.0 \%$ & $42.8 \%$ & $0.0 \%$ & $0.0 \%$ \\
\hline Fig.3 & (c) & $20.0 \%$ & $50.0 \%$ & $0.0 \%$ & $20.0 \%$ \\
\hline
\end{tabular}

Table 2 Comparison of the detection time of the two cases

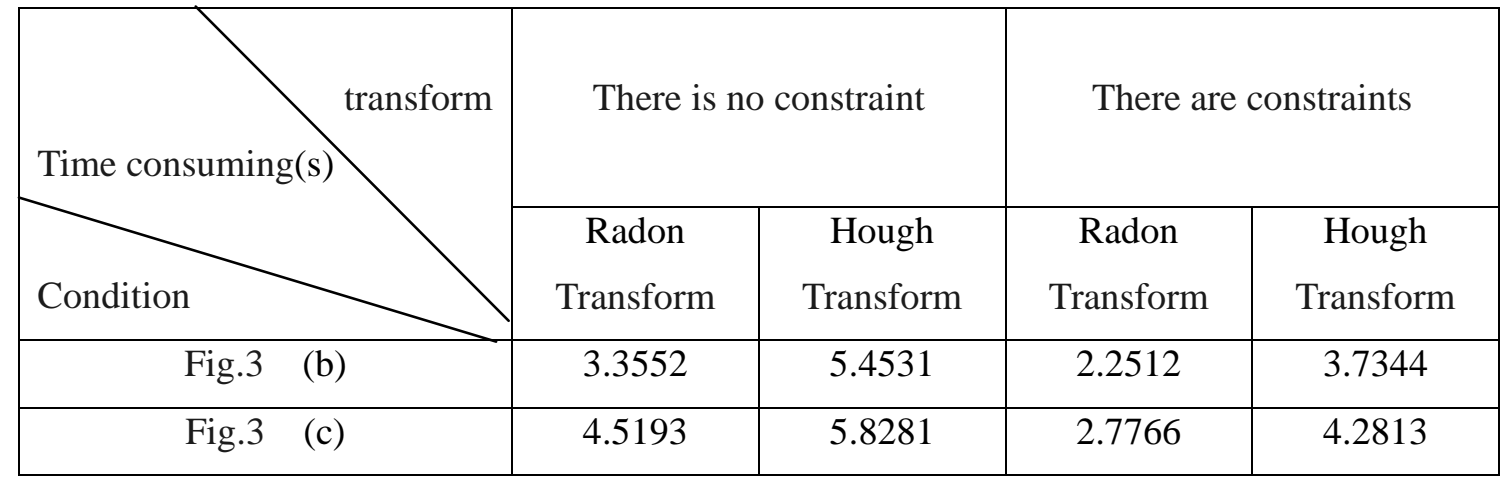

Fig.7 and Fig. 8 show power line detection results of Radon transform and Hough transform using prior knowledge respectively, the effect of power line extraction is improved. Although some 
interference is not excluded after the constraint, the false detection rate is significantly reduced, as shown in Table 1. At the same time, the method also reduces the computation time, and the time is recorded in Table 2. Experiments show that this method can not only greatly improve the circumstance of the wrong extraction of the power line, but also improve the time efficiency of power line recognition.

\section{References}

[1]Liu Yuanjing. Overhead line surrounding environment analysis [D] based on image processing. Wuhan University,2017

[2]Li Cailing, Feng Zhaohui, Deng Xiaojing etc. Power line extraction method in complex terrain background [J]. Computer engineering and Application.2016(22): 198-202.

[3]Chen Y, Li Y, Zhang $\mathrm{H}$, et al. Automatic power line extraction from high resolution remote sensing imagery based on an improved Radon transform[J]. Pattern Recognition. 2016, 49: 174-186.

[4]Zhao Zhenbing, Wang Qin, Gao Qiang. Power line image analysis and extraction [J] using improved phase congruency detection method. High voltage technology.2011(08): 2004-2009.

[5] Zhao Like, Song Weidong, Wang Jingxue. Freeman chain code priority linear extraction algorithm [J]. Journal of Wuhan University (Information Science Edition).2014(01): 42-46.

[6] Von Gioi R G, Jakubowicz J, Morel J M, et al. LSD: A fast line segment detector with a false detection control [J]. IEEE Transactions on Pattern Analysis and Machine Intelligence, 2010, 32(4): 722-732. 